\section{Analysis of an Adaptive Algorithm for Unbiased Multipath Time Delay}

\section{Estimation}

H. C. SO, Member, IEEE

City Unviersity of Hong Kong

The multipath equalization time delay estimator (METDE) provides an adaptive approach for estimating the difference in arrival times of a signal received at spatially separated sensors as well as the multipath channel characteristics. However, the parameter estimates of the METDE are biased in the presence of noise. The METDE algorithm is improved for unbiased parameter estimation via minimizing a modified cost function. Convergence behaviors and variances of the system variables of the amended method are derived. Computer simulations are included to corroborate the theoretical analysis and to evaluate the adaptive multipath delay estimation performance of the proposed algorithm.

Manuscript received December 22, 2000; revised January 11 and November 11, 2002; released for publication April 4, 2003.

IEEE Log No. T-AES/39/3/818480.

Refereeing of this contribution was handled by J. L. Leva.

This work was supported by a grant from the Research Grants Council of the Hong Kong Special Administrative Region, China (Project No. CityU 1119/01E).

Author's address: Dept. of Computer Engineering and Information Technology, City University of Hong Kong, Tat Chee Avenue, Kowloon, Hong Kong, E-mail: (ithcso@ cityu.edu.hk).

\section{INTRODUCTION}

Time delay estimation between signals received at two spatially separated sensors has many important applications such as transmitter linearization [1], synchronization in communication systems [2], speech enhancement, determination of the centre of earthquakes, and source localization in sonar and radio systems [3, 4]. The presence of unknown multipath propagation, which is often encountered in practical situations, has made the task of delay estimation very difficult. For example, acoustic multipath signals come from bottom bounces or reflections from the ocean surface in sonar [5] while radio multipath signals occur where there are reflections from building or mountains in land mobile communications [6]. If the multipaths are ignored, the delay estimation accuracy based on direct-path-only propagation is degraded. On the other hand, when the multipath structure is utilized, fading effects can be alleviated and significant performance improvement can be achieved $[7,8]$.

Using a real signal model, the received outputs of two separated sensors in the presence of multipath transmissions can be represented as

$$
\begin{aligned}
& x(k)=s(k)+\sum_{i=1}^{M_{1}} \alpha_{1 i} s\left(k-\Delta_{1 i}\right)+n_{1}(k) \\
& y(k)=s(k-D)+\sum_{j=1}^{M_{2}} \alpha_{2 j} s\left(k-\Delta_{2 j}\right)+n_{2}(k)
\end{aligned}
$$

where $s(k)$ is the unknown source signal while $n_{1}(k)$ and $n_{2}(k)$ are the uncorrelated white Gaussian noises with variance $\sigma_{n}^{2}$, which are independent of $s(k)$. Without loss of generality, it is assumed that the sampling period is unity second and $s(k)$ is bandlimited between $0 \mathrm{~Hz}$ and $0.5 \mathrm{~Hz}$. The parameter $D$ denotes the time delay between the two sensor outputs. The multipath transmissions are characterized by the gain factors, $\alpha_{1 i}$ and $\alpha_{2 j}$, as well as the interpath delays, $\Delta_{1 i}$ and $\Delta_{2 j}$, for $M_{1} \geq i \geq 1$ and $M_{2} \geq j \geq 1$, such that $\Delta_{11}<\Delta_{12}<\cdots<\Delta_{1 M_{1}}$ and $\Delta_{21}<\Delta_{22}<\cdots<\Delta_{2 M_{2}}$. Notice that the multipath gains must lie between 0 and 1 while $\Delta_{1 i}$ and $\Delta_{2 j}$ should be larger than zero and $D$, respectively. The integers $M_{1}$ and $M_{2}$ are the numbers of multipaths contained in $x(k)$ and $y(k)$ and we consider that they are known a priori. The task is to estimate $D, \alpha_{1 i}, \alpha_{2 j}$, $\Delta_{1 i}$, and $\Delta_{2 j}$ from $x(k)$ and $y(k)$.

When the time difference of arrival and the multipath parameters are nonstationary due to either relative source/receiver motion or time-varying characteristics of the transmission medium, adaptive estimation is necessary to track them over time. In case of a single sensor with one multipath, Smith and Friedlander [9] suggested two adaptive algorithms for 
continuously tracking the multipath delay and gain. The first technique attempts to search the secondary peak in the autocorrelation function while the second employs inverse filtering. Assuming that there is a multipath in only one of the two sensors, two constrained adaptive configurations were proposed [10] to jointly estimate the differential delay and multipath parameters. Basically, the derivation of the two methods is based on the property that a time-shifted version of a bandlimited signal can be modeled by passing the signal through a finite impulse response (FIR) filter whose coefficients are samples of a sinc function [11]. This idea was extended for the case of one multipath at each sensor and two adaptive approaches for direct parameter estimation, namely multipath cancellation time delay estimator (MCTDE) and multipath equalization time delay estimator (METDE), were developed in [12]. Recently, the METDE algorithm was generalized [13] for $M_{1}>1$ and $M_{2}>1$. However, it is illustrated [13] that the estimated delay and multipath parameters provided by the METDE are biased even at high signal-to-noise ratio (SNR) condition, particularly for the multipath gain estimates. The METDE algorithm is improved to acquire unbiased estimation for all system parameters.

The structure of the METDE is first reviewed in Section II. By examining the mean square error (MSE) function of the METDE, a modified cost function whose global minimum gives the exact values of the time delay and multipath parameters, is developed. A bias-free (BF)-METDE algorithm is then proposed which applies the least mean square (LMS) method [14] to minimize the cost function and all parameter estimates are updated explicitly on a sample-by-sample basis. In Section III, learning characteristics and steady state MSEs of the system parameters are analyzed. Simulation results are presented in Section IV to corroborate the theoretical analyses and to evaluate the multipath delay estimation performance of the proposed approach. Finally, conclusions are drawn in Section V.

\section{BIAS-FREE MULTIPATH TIME DELAY ESTIMATION}

Let $X(z), Y(z), S(z), N_{1}(z)$, and $N_{2}(z)$ be the $Z$ transform of $x(k), y(k), s(k), n_{1}(k)$, and $n_{2}(k)$, respectively. Using the interpolation formula [15], $X(z)$ and $Y(z)$ are given by

$$
\begin{aligned}
& X(z)=S(z) A(z)+N_{1}(z) \\
& Y(z)=S(z) B(z)+N_{2}(z)
\end{aligned}
$$

where

$$
A(z)=1+\sum_{i=1}^{M_{1}} \alpha_{1 i} \sum_{m=-\infty}^{\infty} \operatorname{sinc}\left(m-\Delta_{1 i}\right) z^{-m}
$$

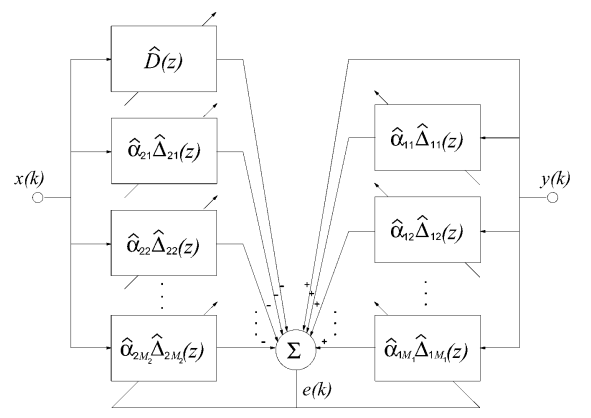

Fig. 1. System block diagram of METDE.

$$
\begin{aligned}
B(z)= & \sum_{m=-\infty}^{\infty} \operatorname{sinc}(m-D) z^{-m} \\
& +\sum_{j=1}^{M_{2}} \alpha_{2 j} \sum_{m=-\infty}^{\infty} \operatorname{sinc}\left(m-\Delta_{2 j}\right) z^{-m}
\end{aligned}
$$

with $\operatorname{sinc}(v) \triangleq \sin (\pi v) /(\pi v)$. When $N_{1}(z)=N_{2}(z)=0$, it is apparent that

$$
X(z) B(z)=Y(z) A(z) .
$$

That is, multiplying $B(z)$ and $A(z)$ to $X(z)$ and $Y(z)$, respectively, the two resultant transfer functions are identical in the absence of noise. The METDE employs this idea of equalization in the time-domain and its system block diagram is depicted in Fig. 1. Basically, it consists of $\left(M_{1}+M_{2}+1\right)$ explicit time delay estimators (ETDEs) [16] and $\left(M_{1}+M_{2}\right)$ adaptive gains. The time-domain error function of the METDE, $e(k)$, has the form

$$
\begin{aligned}
e(k)= & Z^{-1}\left\{Y(z)\left(1+\sum_{i=1}^{M_{1}} \hat{\alpha}_{1 i} \hat{\Delta}_{1 i}(z)\right)\right. \\
& \left.-X(z)\left(\hat{D}(z)+\sum_{j=1}^{M_{2}} \hat{\alpha}_{2 j} \hat{\Delta}_{2 j}(z)\right)\right\} \\
= & y(k)+\sum_{m=-P}^{P} \sum_{i=1}^{M_{1}} \hat{\alpha}_{1 i} \operatorname{sinc}\left(m-\hat{\Delta}_{1 i}\right) y(k-m) \\
& -\sum_{m=-P}^{P}\left(\operatorname{sinc}(m-\hat{D})+\sum_{j=1}^{M_{2}} \hat{\alpha}_{2 j} \operatorname{sinc}\left(m-\hat{\Delta}_{2 j}\right)\right) x(k-m)
\end{aligned}
$$

where $Z^{-1}\{v\}$ represents the inverse $Z$ transform of $v$. The system parameters, $\hat{D}, \hat{\alpha}_{1 i}, \hat{\Delta}_{1 i}, \hat{\alpha}_{2 j}$, and $\hat{\Delta}_{2 j}$, for $M_{1} \geq i \geq 1$ and $M_{2} \geq j \geq 1$, denote the estimates of $D, \alpha_{1 i}, \Delta_{1 i}, \alpha_{2 j}$, and $\Delta_{2 j}$, respectively. The transfer function $\hat{v}(z)$ is defined as $\sum_{m=-P}^{P} \operatorname{sinc}(m-\hat{v}) z^{-m}$ where $P$ is chosen much larger than $\max \left\{\Delta_{1 M_{1}}, \Delta_{2 M_{2}}\right\}$ in order to accurately model the ideal time shift function [11].

Since the signal and noises are independent, squaring and taking expectation of (5) gives the mean 
square value of $e(k)$ :

$$
\begin{gathered}
E\left\{e^{2}(k)\right\} \approx E\left\{\left(\tilde{y}(k)+\sum_{i=1}^{M_{1}} \hat{\alpha}_{1 i} \tilde{y}\left(k-\hat{\Delta}_{1 i}\right)\right.\right. \\
\left.\left.-\tilde{x}(k-\hat{D})-\sum_{j=1}^{M_{2}} \hat{\alpha}_{2 j} \tilde{x}\left(k-\hat{\Delta}_{2 j}\right)\right)^{2}\right\} \\
+E\left\{\left(n_{2}(k)+\sum_{i=1}^{M_{1}} \hat{\alpha}_{1 i} n_{2}\left(k-\hat{\Delta}_{1 i}\right)\right.\right. \\
=E\left\{\left(\tilde{y}(k)+\sum_{i=1}^{M_{1}} \hat{\alpha}_{1 i} \tilde{y}\left(k-\hat{\Delta}_{1 i}\right)\right.\right. \\
\left.\left.-n_{1}(k-\hat{D})-\sum_{j=1}^{M_{2}} \hat{\alpha}_{2 j} n_{1}\left(k-\hat{\Delta}_{2 j}\right)\right)^{2}\right\} \\
\left.\left.-\tilde{x}(k-\hat{D})-\sum_{j=1}^{M_{2}} \hat{\alpha}_{2 j} \tilde{x}\left(k-\hat{\Delta}_{2 j}\right)\right)^{2}\right\}+\sigma_{n}^{2} \gamma
\end{gathered}
$$

where

$$
\begin{aligned}
\gamma= & \sum_{i=0}^{M_{1}} \hat{\alpha}_{1 i}^{2}+2 \sum_{i=0, l>i}^{M_{1}} \hat{\alpha}_{1 i} \hat{\alpha}_{1 l} \operatorname{sinc}\left(\hat{\Delta}_{1 l}-\hat{\Delta}_{1 i}\right)+\sum_{j=0}^{M_{2}} \hat{\alpha}_{2 j}^{2} \\
& +2 \sum_{j=0, l>j}^{M_{2}} \hat{\alpha}_{2 j} \hat{\alpha}_{2 l} \operatorname{sinc}\left(\hat{\Delta}_{2 l}-\hat{\Delta}_{2 j}\right) .
\end{aligned}
$$

The signals $\tilde{x}(k)$ and $\tilde{y}(k)$ represent the noise-free versions of $x(k)$ and $y(k)$, respectively, while $\hat{\alpha}_{10}=$ $\hat{\alpha}_{20}=1, \hat{\Delta}_{10}=0$ and $\hat{\Delta}_{20}=\hat{D}$.

In [13], the METDE algorithm was devised for adaptive multipath time delay estimation by minimizing $E\left\{e^{2}(k)\right\}$ with respect to $\hat{D}, \hat{\alpha}_{1 i}, \hat{\Delta}_{1 i}$, $\hat{\alpha}_{2 j}$, and $\hat{\Delta}_{2 j}$. In a noise-free condition, all parameter estimates provided by this method will be unbiased. However, as illustrated in [13], the METDE cannot give accurate estimates even at high SNR, particularly for the multipath gain parameters. It is because the noise component of $E\left\{e^{2}(k)\right\}$ is also a function of the system variables, as seen from (6). To remove the effect of noise, we propose a modified MSE function, $E\left\{\zeta^{2}(k)\right\}$, which is expressed as

$$
E\left\{\zeta^{2}(k)\right\}=\frac{E\left\{e^{2}(k)\right\}}{\gamma} .
$$

It is easy to prove that the new performance surface $E\left\{\zeta^{2}(k)\right\}$ has the minimum at $\hat{D}=D, \hat{\alpha}_{1 i}=\alpha_{1 i}, \Delta_{1 i}=$ $\hat{\Delta}_{1 i}, \alpha_{2 j}=\hat{\alpha}_{2 j}$, and $\Delta_{2 j}=\hat{\Delta}_{2 j}$. Searching the minimum point is in fact a difficult task because $E\left\{\zeta^{2}(k)\right\}$ is multimodal. However, if we can obtain initial guesses of the delay and multipath parameters such that they correspond to a point on the error surface which is sufficiently close to the global minimum, then simple gradient search approach can be utilized to minimize $E\left\{\zeta^{2}(k)\right\}$.

In our study, the LMS-based MCTDE algorithm [12] is used at the beginning of the adaptation to initialize the system parameters. The MCTDE consists of two adaptive infinite impulse response (IIR) filters for multipath cancellation in each received signal and one adaptive FIR filter for delay estimation. Coarse estimates of $\Delta_{1 i}$ and $\Delta_{2 j}$ are derived from the peak coefficients of the IIR filters, assuming that the multipath delays are resolvable, that is, $\Delta_{1 i+1}-\Delta_{1 i}>$ $1 \mathrm{~s}$ and $\Delta_{2 j+1}-\Delta_{2 j}>1 \mathrm{~s}$. While an initial value of $D$ is deduced from the largest filter weight of the FIR filter. On the other hand, initial estimates of the multipath gains can be arbitrarily selected between 0 and 1 without affecting the global convergence.

After initialization, we employ the LMS method again to minimize $E\left\{\zeta^{2}(k)\right\}$ iteratively so that an unbiased parameter estimation can be achieved in the time-domain BF-METDE. The instantaneous value of $E\left\{\zeta^{2}(k)\right\}, \zeta^{2}(k)$, is given by $\zeta^{2}(k)=e^{2}(k) / \gamma(k)$ where $e(k)$ and $\gamma(k)$ are computed from (5) and (7) using the delay and multipath parameter estimates at time $k$, viz. $\hat{D}(k), \hat{\alpha}_{1 i}(k), \hat{\Delta}_{1 i}(k), \hat{\alpha}_{2 j}(k)$, and $\hat{\Delta}_{2 j}(k)$.

The error gradients in the BF-METDE algorithm are obtained by differentiating $\zeta^{2}(k)$ with respect to the estimated delay and multipath parameters and then multiplying each of the resultant expressions by a positive scaling factor, namely, $\gamma(k) / 2$. The motivation of the latter step is to make the algorithm simpler and easier to analyze, without affecting its unbiasedness. As a result, the LMS updating equations are

$$
\begin{aligned}
& \hat{D}(k+1)=\hat{D}(k)-\mu_{D} e(k) \\
& \quad \times\left(\hat{x}^{\prime}(k-\hat{D}(k))+\frac{e(k)}{\gamma(k)} \sum_{l=1}^{M_{2}} \hat{\alpha}_{2 l}(k) f\left(\hat{\Delta}_{2 l}(k)-\hat{D}(k)\right)\right) \\
& \hat{\alpha}_{1 i}(k+1)=\hat{\alpha}_{1 i}(k)-\mu_{\alpha} e(k) \\
& \quad \times\left(\hat{y}\left(k-\hat{\Delta}_{1 i}(k)\right)-\frac{e(k)}{\gamma(k)} \sum_{l=0}^{M_{1}} \hat{\alpha}_{1 l}(k) \operatorname{sinc}\left(\hat{\Delta}_{1 i}(k)-\hat{\Delta}_{1 l}(k)\right)\right)
\end{aligned}
$$

$$
\begin{aligned}
& \hat{\Delta}_{1 i}(k+1)=\hat{\Delta}_{1 i}(k)+\mu_{\Delta} e(k) \\
& \quad \times\left(\hat{y}^{\prime}\left(k-\hat{\Delta}_{1 i}(k)\right)+\frac{e(k)}{\gamma(k)} \sum_{l=0, l \neq i}^{M_{1}} \hat{\alpha}_{1 l}(k) f\left(\hat{\Delta}_{1 i}(k)-\hat{\Delta}_{1 l}(k)\right)\right)
\end{aligned}
$$

$$
\begin{aligned}
& \hat{\alpha}_{2 j}(k+1)=\hat{\alpha}_{2 j}(k)+\mu_{\alpha} e(k) \\
& \quad \times\left(\hat{x}\left(k-\hat{\Delta}_{2 j}(k)\right)+\frac{e(k)}{\gamma(k)} \sum_{l=0}^{M_{2}} \hat{\alpha}_{2 l}(k) \operatorname{sinc}\left(\hat{\Delta}_{2 i}(k)-\hat{\Delta}_{2 l}(k)\right)\right)
\end{aligned}
$$




$$
\begin{aligned}
& \hat{\Delta}_{2 j}(k+1)=\hat{\Delta}_{2 j}(k)-\mu_{\Delta} e(k) \\
& \quad \times\left(\hat{x}^{\prime}\left(k-\hat{\Delta}_{2 j}(k)\right)-\frac{e(k)}{\gamma(k)} \sum_{l=0, l \neq j}^{M_{2}} \hat{\alpha}_{2 l}(k) f\left(\hat{\Delta}_{2 j}(k)-\hat{\Delta}_{2 l}(k)\right)\right)
\end{aligned}
$$

where $\hat{u}(k-v)=\sum_{m=-P}^{P} \operatorname{sinc}(m-v) u(k-m)$ and $\hat{u}^{\prime}(k-v)=\sum_{m=-P}^{P} f(m-v) u(k-m)$, and $u$ and $v$ represent the signals and delay parameters, respectively. The positive scalars $\mu_{D}, \mu_{\alpha}$, and $\mu_{\Delta}$ are the step sizes of delay, multipath gain, and multipath delay estimates, respectively, and $f(v)=(\cos (\pi v)-$ $\operatorname{sinc}(v)) / v$. The quantities $\hat{\alpha}_{10}(k)$ and $\hat{\alpha}_{20}(k)$ are equal to one while $\hat{\Delta}_{10}(k)=0$ and $\hat{\Delta}_{20}(k)=\hat{D}(k)$. It is noteworthy that if $\gamma(k) \rightarrow \infty$, (9)-(13) will become the METDE algorithm [13].

To reduce computational complexity, values of the sinc and $f$ function are retrieved through table look-up operations [16]. It can be shown that $\left(1+M_{1}+M_{2}\right)(2 P+1)-1$ additions and $(3+$ $\left.M_{1}+M_{2}\right)(2 P+1)$ multiplications, and $M_{1}\left(M_{1}+2\right)+$ $M_{2}\left(M_{2}+2\right)+1$ additions and $\left(M_{1}+1\right)^{2}+\left(M_{2}+1\right)^{2}+2$ multiplications are required to compute $e(k)$ and $\gamma(k)$, respectively, for each sampling interval. Adjustment for $\hat{D}(k)$ or $\hat{\Delta}_{2 j}(k)$ and $\hat{\Delta}_{1 i}(k)$ need further $(6 P+$ $\left.2 M_{2}+3\right)$ additions and $\left(4 P+M_{2}+5\right)$ multiplications, and $\left(6 P+2 M_{1}+3\right)$ additions and $\left(4 P+M_{1}+5\right)$ multiplications. While $\left(2 P+2 M_{m}+3\right)$ additions and $\left(2 P+M_{m}+5\right)$ multiplications, $m=1$ and 2 , are involved in calculating $\hat{\alpha}_{1 i}(k)$ and $\hat{\alpha}_{2 j}(k)$, respectively. We see that when $P \gg \max \left\{M_{1}, M_{2}\right\}$, the total amount of computation is roughly proportional to the number of multipaths and $P$.

\section{PERFORMANCE ANALYSIS OF BF-METDE}

For brevity, the learning rates as well as the steady state MSEs of the BF-METDE parameter estimates when $s(k)$ is a white process with variance $\sigma_{s}^{2}$ are examined in this section. Taking the expected values of (9)-(13), the convergence behaviors of the delay and multipath parameter estimates are derived as (see Appendix A)

$$
\begin{gathered}
E\{\hat{D}(k)\} \approx D+(\hat{D}(0)-D)\left(1-\frac{1}{3} \mu_{D} \sigma_{s}^{2} \pi^{2}\left(1+\sum_{i=1}^{M_{1}} \alpha_{1 i}^{2}\right)\right)^{k} \\
E\left\{\hat{\alpha}_{1 i}(k)\right\} \approx \alpha_{1 i}+\left(\hat{\alpha}_{1 i}(0)-\alpha_{1 i}\right)\left(1-\mu_{\alpha} \sigma_{y}^{2}\right)^{k} \\
E\left\{\hat{\Delta}_{1 i}(k)\right\} \approx \Delta_{1 i}+\left(\hat{\Delta}_{1 i}(0)-\Delta_{1 i}\right) \\
\times\left(1-\frac{1}{3} \mu_{\Delta} \sigma_{s}^{2} \pi^{2} \alpha_{1 i}\left(1+\sum_{j=1}^{M_{2}} \alpha_{2 j}^{2}\right)\right)^{k} \\
E\left\{\hat{\alpha}_{2 j}(k)\right\} \approx
\end{gathered}
$$

$$
\begin{aligned}
E\left\{\hat{\Delta}_{2 j}(k)\right\} \approx & \Delta_{2 j}+\left(\hat{\Delta}_{2 j}(0)-\Delta_{2 j}\right) \\
& \times\left(1-\frac{1}{3} \mu_{\Delta} \sigma_{s}^{2} \pi^{2} \alpha_{2 j}\left(1+\sum_{i=1}^{M_{1}} \alpha_{1 i}^{2}\right)\right)^{k}
\end{aligned}
$$

where $\sigma_{x}^{2}$ and $\sigma_{y}^{2}$ denote the power of $x(k)$ and $y(k)$, respectively, and they are given by $\sigma_{x}^{2}=\sigma_{s}^{2}\left(\sum_{i=0}^{M_{1}} \alpha_{1 i}^{2}+\right.$ $\left.2 \sum_{i=0, l>i}^{M_{1}} \alpha_{1 i} \alpha_{1 l} \operatorname{sinc}\left(\Delta_{1 l}-\Delta_{1 i}\right)\right)+\sigma_{n}^{2}$ and $\sigma_{y}^{2}=$ $\sigma_{s}^{2}\left(\sum_{j=0}^{M_{2}} \alpha_{2 j}^{2}+2 \sum_{j=0, l>j}^{M_{2}} \alpha_{2 j} \alpha_{2 l} \operatorname{sinc}\left(\Delta_{2 l}-\Delta_{2 j}\right)\right)+$ $\sigma_{n}^{2}$. The variables $\hat{D}(0), \hat{\alpha}_{1 i}(0), \hat{\Delta}_{1 i}(0), \hat{\alpha}_{2 j}(0)$ and $\hat{\Delta}_{2 j}(0)$ represent the initial estimates of the delay and multipath parameters. The convergence of the algorithm is guaranteed as long as the magnitudes of the terms raised to the $k$ th power in (14)-(18) are less than unity. As a result, when $\mu_{D}$ and $\mu_{\Delta}$ are bounded by $\min \left\{6 /\left(\sigma_{s}^{2} \pi^{2}\left(1+M_{1}\right)\right), 6 /\left(\sigma_{s}^{2} \pi^{2}\left(1+M_{2}\right)\right)\right\}$ and $0<\mu_{\alpha}<\min \left\{2 / \sigma_{x}^{2}, 2 / \sigma_{y}^{2}\right\}$, the algorithm will provide unbiased estimates of $D, \alpha_{1 i}, \alpha_{2 j}, \Delta_{1 i}$, and $\Delta_{2 j}$ upon convergence. We also observe that the time constants of the delay and multipath parameter estimates are different and they depend on the actual values of the interpath delays and multipath gains.

The steady state MSEs of $\hat{D}(k), \hat{\alpha}_{1 i}(k), \hat{\Delta}_{1 i}(k)$, $\hat{\alpha}_{2 j}(k)$ and $\hat{\Delta}_{2 j}(k)$, denoted by $\operatorname{var}(\hat{D}), \operatorname{var}\left(\hat{\alpha}_{1 i}\right)$, $\operatorname{var}\left(\hat{\Delta}_{1 i}\right), \operatorname{var}\left(\hat{\alpha}_{2 j}\right)$ and $\operatorname{var}\left(\hat{\Delta}_{2 j}\right)$, respectively, are given by (see Appendix B)

$$
\begin{gathered}
\operatorname{var}(\hat{D}) \triangleq \lim _{k \rightarrow \infty} E\left\{(\hat{D}(k)-D)^{2}\right\} \approx \frac{\mu_{D} \sigma_{a}^{2}}{\mathrm{SNR}} \\
\operatorname{var}\left(\hat{\alpha}_{1 i}\right) \triangleq \lim _{k \rightarrow \infty} E\left\{\left(\hat{\alpha}_{i 1}(k)-\alpha_{i 1}\right)^{2}\right\} \approx \frac{\mu_{\alpha} \sigma_{a}^{2}}{\mathrm{SNR}} \\
\operatorname{var}\left(\hat{\Delta}_{1 i}\right) \triangleq \lim _{k \rightarrow \infty} E\left\{\left(\hat{\Delta}_{1 i}(k)-\Delta_{1 i}\right)^{2}\right\} \approx \frac{\mu_{\Delta} \sigma_{a}^{2}}{\alpha_{1 i} \mathrm{SNR}} \\
\operatorname{var}\left(\hat{\alpha}_{2 j}\right) \triangleq \lim _{k \rightarrow \infty} E\left\{\left(\hat{\alpha}_{2 j}(k)-\alpha_{2 j}\right)^{2}\right\} \approx \frac{\mu_{\alpha} \sigma_{a}^{2}}{\mathrm{SNR}} \\
\operatorname{var}\left(\hat{\Delta}_{2 j}\right) \triangleq \lim _{k \rightarrow \infty} E\left\{\left(\hat{\Delta}_{2 j}(k)-\Delta_{2 j}\right)^{2}\right\} \approx \frac{\mu_{\Delta} \sigma_{a}^{2}}{\alpha_{2 j} \mathrm{SNR}}
\end{gathered}
$$

where $\sigma_{a}^{2}=\left(\sigma_{x}^{2}+\sigma_{y}^{2}-2 \sigma_{n}^{2}\right) / 2$ is the mean signal power at the two sensors and $\mathrm{SNR}=\sigma_{s}^{2} / \sigma_{n}^{2}$. We observe that the variances of the parameter estimates increase with the values of the step sizes and $\sigma_{a}^{2}$, and decrease with SNR. In particular, $\operatorname{var}\left(\hat{\Delta}_{1 i}\right)$ and $\operatorname{var}\left(\hat{\Delta}_{2 j}\right)$ decrease as $\alpha_{1 i}$ and $\alpha_{2 j}$ increase, respectively. Notice that since independence of the parameter estimates has been assumed at each iteration in the above derivations, their rates of convergence may differ from (14)-(18) in actual circumstances. Furthermore, the steady state variances will be greater than the theoretical values and have finite values even in the absence of noise. 
TABLE I

Parameter Estimates and MSEs of BF-METDE and METDE for $M_{1}=M_{2}=1$ at $\mathrm{SNR}=10 \mathrm{~dB}$

\begin{tabular}{cc|ccc|cc}
\hline \hline & \multicolumn{3}{|c|}{ BF-METDE } & \multicolumn{2}{c}{ METDE } \\
\cline { 3 - 7 } & $\begin{array}{c}\text { Actual } \\
\text { value }\end{array}$ & $\begin{array}{c}\text { Measured } \\
\text { Mean }\end{array}$ & $\begin{array}{c}\text { Theoretical } \\
\text { MSE }\end{array}$ & $\begin{array}{c}\text { Measured } \\
\text { MSE }\end{array}$ & $\begin{array}{c}\text { Measured } \\
\text { Mean }\end{array}$ & $\begin{array}{c}\text { Measured } \\
\text { MSE }\end{array}$ \\
\hline$\hat{D}(k)$ & $0.5 \mathrm{~s}$ & $0.501 \mathrm{~s}$ & $4.381 \times 10^{-4} \mathrm{~s}^{2}$ & $6.358 \times 10^{-4} \mathrm{~s}^{2}$ & $0.534 \mathrm{~s}$ & $1.746 \times 10^{-3} \mathrm{~s}^{2}$ \\
$\hat{\alpha}_{11}(k)$ & 0.8 & 0.802 & $8.763 \times 10^{-4}$ & $1.241 \times 10^{-3}$ & 0.706 & $1.004 \times 10^{-2}$ \\
$\hat{\Delta}_{11}(k)$ & $1.6 \mathrm{~s}$ & $1.603 \mathrm{~s}$ & $5.477 \times 10^{-4} \mathrm{~s}^{2}$ & $7.784 \times 10^{-4} \mathrm{~s}^{2}$ & $1.561 \mathrm{~s}$ & $2.228 \times 10^{-3} \mathrm{~s}^{2}$ \\
$\hat{\alpha}_{21}(k)$ & 0.7 & 0.704 & $8.763 \times 10^{-4}$ & $1.294 \times 10^{-3}$ & 0.588 & $1.371 \times 10^{-2}$ \\
$\hat{\Delta}_{21}(k)$ & $3.3 \mathrm{~s}$ & $3.294 \mathrm{~s}$ & $6.259 \times 10^{-4} \mathrm{~s}^{2}$ & $7.828 \times 10^{-4} \mathrm{~s}^{2}$ & $3.397 \mathrm{~s}$ & $1.012 \times 10^{-2} \mathrm{~s}^{2}$ \\
\hline
\end{tabular}

\section{SIMULATION RESULTS}

Computer simulations were conducted to evaluate the performance of the proposed algorithm for time delay estimation in the presence of multipath transmissions. The signal $s(k)$ as well as the noises $n_{1}(k)$ and $n_{2}(k)$ were independent zero-mean white Gaussian processes. The power of $s(k)$ was fixed to unity and the random noise sequences were scaled to obtain the required SNR. We assumed that $D \in$ $(-1 \mathrm{~s}, 1 \mathrm{~s})$ and the values of the multipath delays were less than $8 \mathrm{~s}$. In order to allow for acceptable delay modeling error, $P$ was chosen to be 15 . In our experiments, we freely adapted the MCTDE at the beginning for 600 iterations to determine the initial estimates of $D, \Delta_{1 i}$, and $\Delta_{2 j}$. While the values of $\hat{\alpha}_{1 i}(0)$ and $\hat{\alpha}_{2 j}(0)$ were arbitrarily selected to be 0.5 . The step size $\mu_{\alpha}$ had a value of 0.006 and $\mu_{D}=\mu_{\Delta}=$ 0.0003 were used. All simulation results provided were averages of 100 independent runs.

Figs. 2 to 6 show the learning behaviors for $\hat{D}(k)$, $\hat{\alpha}_{11}(k), \hat{\Delta}_{11}(k), \hat{\alpha}_{21}(k)$, and $\hat{\Delta}_{21}(k)$ of the BF-METDE and METDE at an SNR of $10 \mathrm{~dB}$. Two multipath conditions, namely, $M_{1}=M_{2}=1$ and $M_{1}=M_{2}=2$ were tried. For $M_{1}=M_{2}=1$, the actual values of the system parameters were given as follows, $D=0.5 \mathrm{~s}$, $\alpha_{11}=0.8, \Delta_{11}=1.6 \mathrm{~s}, \alpha_{21}=0.7$, and $\Delta_{21}=3.3 \mathrm{~s}$. It can be seen that for the modified algorithm, the delay estimate reached steady state in about 1200 iterations while the multipath variables $\hat{\alpha}_{11}(k), \hat{\Delta}_{11}(k)$, $\hat{\alpha}_{21}(k), \hat{\Delta}_{21}(k)$ converged at approximately the 1000th, 1400 th, 1800th, and 1500th iteration, respectively. We also observe that the convergence dynamics of the delay and multipath parameter estimates of the BF-METDE generally agreed with their expected trajectories. On the other hand, the METDE provided similar learning speed but its parameter estimates were biased in the presence of noise. Table I tabulates the steady state parameter estimates and MSEs of the BF-METDE and METDE. It can be seen that the mean estimates of all BF-METDE variables approached the desired values, which illustrates the unbiasedness of the algorithm. Furthermore, all measured MSEs agreed with their predicted values of (19)-(23), although the latter was always smaller. It

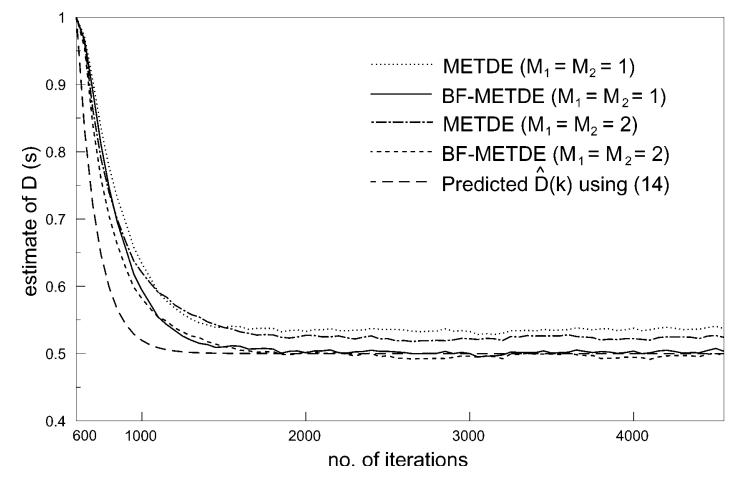

Fig. 2. Estimates of $D$ using METDE and BF-METDE.

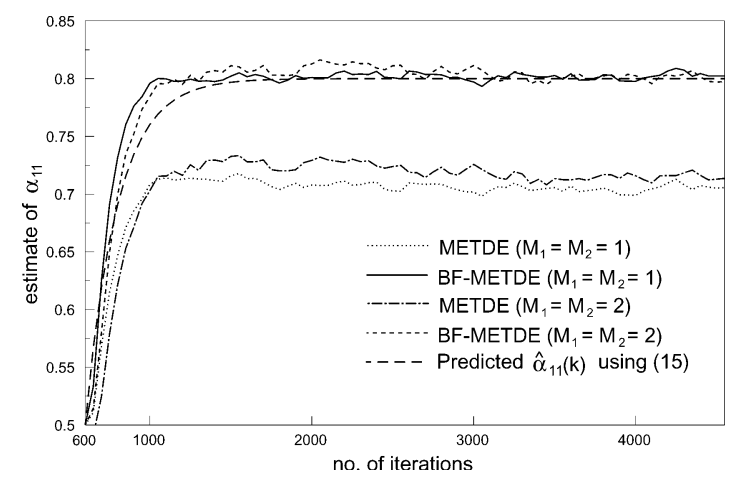

Fig. 3. Estimates of $\alpha_{11}$ using METDE and BF-METDE.

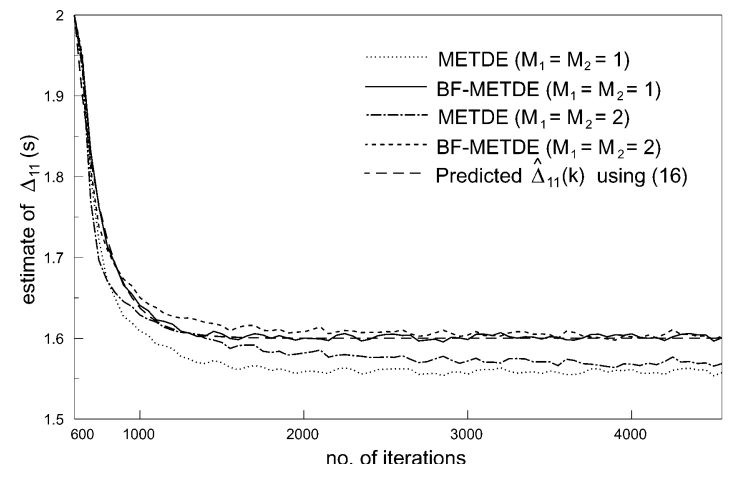

Fig. 4. Estimates of $\Delta_{11}$ using METDE and BF-METDE.

is because in the theoretical derivation of any one of the parameter estimates, we assume that the remaining $2\left(M_{1}+M_{2}\right)$ variables have converged to their desired values. On the contrary, the METDE had much larger 
TABLE II

Parameter Estimates and MSEs of BF-METDE and METDE for $M_{1}=M_{2}=2$ at $\mathrm{SNR}=10 \mathrm{~dB}$

\begin{tabular}{cc|ccc|cc}
\hline \hline & & \multicolumn{3}{|c|}{ BF-METDE } & \multicolumn{2}{c}{ METDE } \\
\cline { 3 - 7 } & $\begin{array}{c}\text { Actual } \\
\text { value }\end{array}$ & $\begin{array}{c}\text { Measured } \\
\text { Mean }\end{array}$ & $\begin{array}{c}\text { Theoretical } \\
\text { MSE }\end{array}$ & $\begin{array}{c}\text { Measured } \\
\text { MSE }\end{array}$ & $\begin{array}{c}\text { Measured } \\
\text { Mean }\end{array}$ & $\begin{array}{c}\text { Measured } \\
\text { MSE }\end{array}$ \\
\hline$\hat{D}(k)$ & $0.5 \mathrm{~s}$ & $0.497 \mathrm{~s}$ & $4.365 \times 10^{-4} \mathrm{~s}^{2}$ & $6.379 \times 10^{-4} \mathrm{~s}^{2}$ & $0.525 \mathrm{~s}$ & $1.203 \times 10^{-3} \mathrm{~s}^{2}$ \\
$\hat{\alpha}_{11}(k)$ & 0.8 & 0.799 & $8.729 \times 10^{-4}$ & $1.460 \times 10^{-3}$ & 0.714 & $8.657 \times 10^{-3}$ \\
$\hat{\Delta}_{11}(k)$ & $1.6 \mathrm{~s}$ & $1.600 \mathrm{~s}$ & $5.456 \times 10^{-4} \mathrm{~s}^{2}$ & $8.881 \times 10^{-4} \mathrm{~s}^{2}$ & $1.567 \mathrm{~s}$ & $1.869 \times 10^{-3} \mathrm{~s}^{2}$ \\
$\hat{\alpha}_{21}(k)$ & 0.7 & 0.705 & $8.729 \times 10^{-4}$ & $1.567 \times 10^{-3}$ & 0.596 & $1.246 \times 10^{-2}$ \\
$\hat{\Delta}_{21}(k)$ & $3.3 \mathrm{~s}$ & $3.303 \mathrm{~s}$ & $6.235 \times 10^{-4} \mathrm{~s}^{2}$ & $1.144 \times 10^{-3} \mathrm{~s}^{2}$ & $3.405 \mathrm{~s}$ & $1.198 \times 10^{-2} \mathrm{~s}^{2}$ \\
$\hat{\alpha}_{12}(k)$ & 0.4 & 0.407 & $8.729 \times 10^{-4}$ & $1.549 \times 10^{-3}$ & 0.397 & $1.238 \times 10^{-3}$ \\
$\hat{\Delta}_{12}(k)$ & $5.1 \mathrm{~s}$ & $5.095 \mathrm{~s}$ & $1.091 \times 10^{-3} \mathrm{~s}^{2}$ & $1.678 \times 10^{-3} \mathrm{~s}^{2}$ & $5.164 \mathrm{~s}$ & $5.490 \times 10^{-3} \mathrm{~s}^{2}$ \\
$\hat{\alpha}_{22}(k)$ & 0.3 & 0.300 & $8.729 \times 10^{-4}$ & $1.586 \times 10^{-3}$ & 0.289 & $1.520 \times 10^{-3}$ \\
$\hat{\Delta}_{22}(k)$ & $7.2 \mathrm{~s}$ & $7.213 \mathrm{~s}$ & $1.455 \times 10^{-3} \mathrm{~s}^{2}$ & $2.153 \times 10^{-3} \mathrm{~s}^{2}$ & $7.186 \mathrm{~s}$ & $1.820 \times 10^{-3} \mathrm{~s}^{2}$ \\
\hline
\end{tabular}

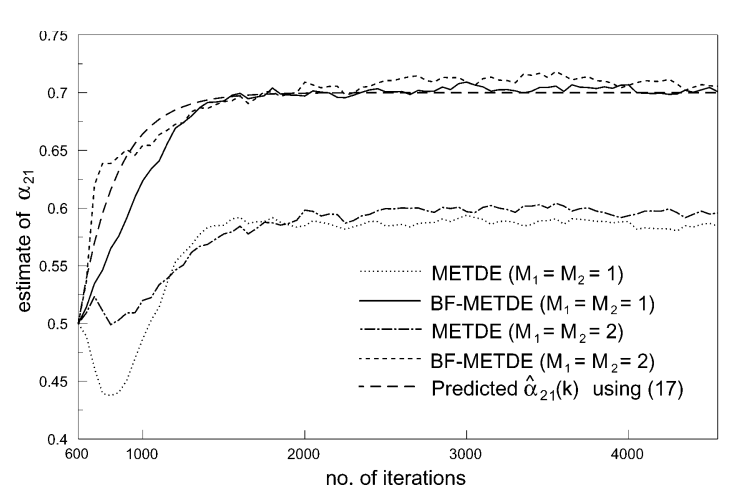

Fig. 5. Estimates of $\alpha_{21}$ using METDE and BF-METDE.

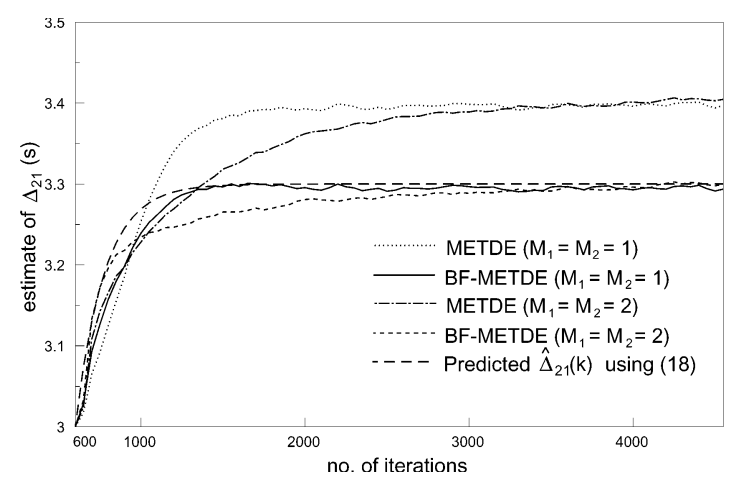

Fig. 6. Estimates of $\Delta_{21}$ using METDE and BF-METDE.

MSEs due to its biased estimates. The variances of the METDE could be approximated by subtracting the squared bias of the parameter estimates from the corresponding MSEs, and we found these values were close to the MSEs of the BF-METDE. This implies that there is no bias/variance tradeoff in the BF-METDE.

For $M_{1}=M_{2}=2$, the values of the additional parameters were $\alpha_{12}=0.4, \Delta_{12}=5.1 \mathrm{~s}, \alpha_{21}=$ 0.3 , and $\Delta_{22}=7.2$ s. From Figs. 2 to 6 again, we observe that the learning trajectories of the METDE and BF-METDE parameters for $M_{1}=M_{2}=2$ were similar to those of $M_{1}=M_{2}=1$. Although not shown, the learning trajectories of the other BF-METDE parameters also agreed with (15)-(18). The steady state parameter estimates and MSEs of the BF-METDE and METDE for this multipath condition are shown in Table II. It is seen that the BF-METDE still provided unbiased estimates for all system parameters. Moreover, its measured MSEs conformed to the theoretical calculations but the degree of agreement was poorer than the case of $M_{1}=M_{2}=1$, which is expected since the approximations of (19)-(23) weaken when $M_{1}$ and/or $M_{2}$ increase. On the other hand, the METDE was inferior to the BF-METDE although it provided comparable performance for $\hat{\alpha}_{12}(k), \hat{\alpha}_{22}(k)$, and $\hat{\Delta}_{22}(k)$ because the biases in these parameters were obscure. From Tables I and II, we notice that the MSEs of both methods do not necessarily increase as the number of multipaths increases but they should depend on $D$ and the multipath parameters.

\section{CONCLUSIONS}

The METDE algorithm is improved for unbiased estimation of the time delay between signals received at two separated receivers and the channel parameters in the presence of multipath transmissions. The estimation procedure consists of two steps. Coarse estimates of the system parameters are first obtained by using the MCTDE system. The enhanced METDE algorithm is then used to attain unbiased and direct estimates of the delay and multipath parameters through minimization of a modified cost function. Learning behaviors and MSEs of the estimated system parameters are derived and verified by computer simulations.

\section{APPENDIX A}

In analyzing any one of the parameter estimates, we consider sufficiently small step sizes and the 
remaining $2\left(M_{1}+M_{2}\right)$ variables have converged to their desired values, so that the derivations can be greatly simplified. For example, it is assumed that $\hat{\alpha}_{1 i}(k) \rightarrow \alpha_{1 i}, \hat{\Delta}_{1 i}(k) \rightarrow \Delta_{1 i}, \hat{\alpha}_{2 j}(k) \rightarrow \alpha_{2 j}$ and $\hat{\Delta}_{2 j}(k) \rightarrow$ $\Delta_{2 j}$ when calculating the statistical behavior of the delay estimate. We also assume coarse estimates of the system parameters that warrant global convergence have been obtained through proper initialization [12] and $P$ is chosen sufficiently large such that delay modeling error can be neglected. As a result, the error function in calculating the learning behavior of $\hat{D}(k)$ is

$$
\begin{aligned}
e(k) \approx & \sum_{i=0}^{M_{1}} \alpha_{1 i}\left(s\left(k-\Delta_{1 i}-D\right)-s\left(k-\Delta_{1 i}-\hat{D}(k)\right)\right) \\
& +\sum_{i=0}^{M_{1}} \alpha_{1 i} n_{2}\left(k-\Delta_{1 i}\right)-\sum_{j=0}^{M_{2}} \alpha_{2 j} n_{1}\left(k-\Delta_{2 j}\right) .
\end{aligned}
$$

Employing (24) and assuming the interpath delays are highly resolvable, the expected value of $e(k) \hat{x}^{\prime}(k-\hat{D}(k))$ is

$$
\begin{aligned}
& E\left\{e(k) \hat{x}^{\prime}(k-\hat{D}(k))\right\} \\
& \begin{array}{c}
\sum_{i=0}^{M_{1}} \sum_{j=0}^{M_{1}} \sum_{m=-P}^{P} \alpha_{1 i} \alpha_{1 j} E\left\{\left(s\left(k-\Delta_{1 i}-D\right)-s\left(k-\Delta_{1 i}-\hat{D}(k)\right)\right)\right. \\
\left.\quad \times s\left(k-\Delta_{1 j}-m\right) f(m-\hat{D}(k))\right\}
\end{array} \\
& -\sum_{j=0}^{M_{2}} \sum_{m=-P}^{P} \alpha_{2 j} E\left\{n_{1}\left(k-\Delta_{2 j}\right) n_{1}(k-m) f(m-\hat{D}(k))\right\} \\
& \approx \sum_{i=0}^{M_{1}} \sum_{m=-P}^{P} \alpha_{1 i}^{2} E\left\{\left(s\left(k-\Delta_{1 i}-D\right)-s\left(k-\Delta_{1 i}-\hat{D}(k)\right)\right)\right. \\
& \quad-\sigma_{n}^{2} \sum_{j=0}^{M_{2}} \sum_{m=-P}^{P} \alpha_{2 j} E\left\{\operatorname{sinc}\left(\Delta_{2 j}-m\right) f(m-\hat{D}(k))\right\} \\
& \approx \sigma_{s}^{2} \sum_{i=0}^{M_{1}} \sum_{m=-P}^{P} \alpha_{1 i}^{2} E\{(\operatorname{sinc}(m-D)-\operatorname{sinc}(m-\hat{D}(k)) \\
& \quad \times f(m-\hat{D}(k))\} \\
& \quad-\sigma_{n}^{2} \sum_{j=0}^{M_{2}} \alpha_{2 j} E\left\{f\left(\Delta_{2 j}-\hat{D}(k)\right)\right\} \\
& \approx \sigma_{s}^{2} \sum_{i=0}^{M_{1}} \alpha_{1 i}^{2} E\left\{(f(D-\hat{D}(k))\}-\sigma_{n}^{2} \sum_{j=0}^{M_{2}} \alpha_{2 j} E\left\{f\left(\Delta_{2 j}-\hat{D}(k)\right)\right\}\right.
\end{aligned}
$$

with the use of $f(0)=0$ and $E\{w(k-u) w(k-v)\}$ $=\sigma_{w}^{2} \operatorname{sinc}(u-v)$ where $w$ is a white process with variance $\sigma_{w}^{2}$. To derive (14), we also need the following term:

$$
\begin{aligned}
E\left\{\frac{e^{2}(k)}{\gamma(k)} \sum_{l=1}^{M_{2}} \alpha_{2 l} f\left(\Delta_{2 l}-\hat{D}(k)\right)\right\} & \\
\approx & \frac{E\left\{e^{2}(k)\right\}}{E\{\gamma(k)\}} E\left\{\sum_{l=1}^{M_{2}} \alpha_{2 l} f\left(\Delta_{2 l}-\hat{D}(k)\right)\right\} \\
= & \sigma_{n}^{2} \sum_{l=1}^{M_{2}} \alpha_{2 l} E\left\{f\left(\Delta_{2 l}-\hat{D}(k)\right)\right\} .
\end{aligned}
$$

From (9), (25) and (26), we have

$$
\begin{aligned}
& E\{\hat{D}(k+1)\} \\
& \quad \approx E\{\hat{D}(k)\}-\mu_{D} \sigma_{s}^{2}\left(1+\sum_{i=1}^{M_{1}} \alpha_{1 i}^{2}\right) E\{(f(D-\hat{D}(k))\} .
\end{aligned}
$$

Expand $f(D-\hat{D}(k))$ up to the first-order term using Taylor's series and solve for (27); (14) is obtained. In a similar manner, (15)-(18) can be derived.

\section{APPENDIX B}

The steady state MSE of $\hat{D}(k)$ is derived as follows. Subtracting $D$ from both sides of (9), squaring both sides, taking expectation and then considering $k \rightarrow \infty$ yields

$$
\begin{gathered}
2 \mu_{D} \lim _{k \rightarrow \infty} E\left\{( \hat { D } ( k ) - D ) e ( k ) \left(\hat{x}^{\prime}(k-\hat{D}(k))+\frac{e(k)}{\gamma(k)} \sum_{l=1}^{M_{2}} \hat{\alpha}_{2 l}(k)\right.\right. \\
\left.\left.\times f\left(\hat{\Delta}_{2 l}(k)-\hat{D}(k)\right)\right)\right\} \\
=\mu_{D}^{2} \lim _{k \rightarrow \infty} E\left\{e ^ { 2 } ( k ) \left(\hat{x}^{\prime}(k-\hat{D}(k))+\frac{e(k)}{\gamma(k)} \sum_{l=1}^{M_{2}} \hat{\alpha}_{2 l}(k)\right.\right. \\
\left.\left.\times f\left(\hat{\Delta}_{2 l}(k)-\hat{D}(k)\right)\right)^{2}\right\} .
\end{gathered}
$$

Using the results of Appendix A, the LHS of (28) is approximated as

$$
\begin{aligned}
& 2 \mu_{D} \sigma_{s}^{2}\left(1+\sum_{i=1}^{M_{1}} \alpha_{1 i}^{2}\right) \lim _{k \rightarrow \infty} E\{(\hat{D}(k)-D) f(D-\hat{D}(k))\} \\
& \approx \frac{2}{3} \pi^{2} \mu_{D} \sigma_{s}^{2}\left(1+\sum_{i=1}^{M_{1}} \alpha_{1 i}^{2}\right) \operatorname{var}(\hat{D}) .
\end{aligned}
$$

Let $\hat{x}_{s}^{\prime}(k-\hat{D}(k))$ be the noise-free component of $\hat{x}^{\prime}(k-\hat{D}(k))$. With the use of $\sum_{m=-\infty}^{\infty} f^{2}(m-D)=$ 
$\pi^{2} / 3$, the RHS of (28) is calculated as

$$
\begin{aligned}
& \mu_{D}^{2} \lim _{k \rightarrow \infty} E\left\{e ^ { 2 } ( k ) \left(\hat{x}^{\prime}(k-\hat{D}(k))+\frac{e(k)}{\gamma(k)} \sum_{l=1}^{M_{2}} \hat{\alpha}_{2 l}(k)\right.\right. \\
& \left.\left.\times f\left(\hat{\Delta}_{2 l}(k)-\hat{D}(k)\right)\right)^{2}\right\} \\
& \approx \mu_{D}^{2} \lim _{k \rightarrow \infty} E\left\{e^{2}(k)\right\} \cdot E\left\{\left(\hat{x}_{s}^{\prime}(k-D)\right)^{2}\right\} \\
& \approx \mu_{D}^{2} \sigma_{n}^{2} \lim _{k \rightarrow \infty} E\{\gamma(k)\} \\
& \cdot E\left\{\left(\sum_{m=-P}^{P} f(m-D) \sum_{i=0}^{M_{1}} \alpha_{1 i} s\left(k-m-\Delta_{1 i}\right)\right)^{2}\right\} \\
& =\mu_{D}^{2} \sigma_{n}^{2} \cdot \frac{2 \sigma_{a}^{2}}{\sigma_{s}^{2}} \cdot \sum_{m=-P}^{P} \sum_{n=-P}^{P} f(m-D) f(n-D) \\
& \cdot E\left\{\sum_{i=0}^{M_{1}} \sum_{j=0}^{M_{1}} \alpha_{1 i} \alpha_{1 j} s\left(k-m-\Delta_{1 i}\right) s\left(k-n-\Delta_{1 j}\right)\right\} \\
& \approx \mu_{D}^{2} \frac{2 \sigma_{a}^{2}}{\mathrm{SNR}} \sum_{m=-P}^{P} f^{2}(m-D) \\
& \cdot \sum_{i=0}^{M_{1}} \alpha_{1 i}^{2} E\left\{s^{2}\left(k-m-\Delta_{1 i}\right)\right\} \\
& \approx \mu_{D}^{2} \frac{2 \sigma_{a}^{2}}{\mathrm{SNR}} \cdot \frac{\pi^{2}}{3} \cdot \sigma_{s}^{2} \sum_{i=0}^{M_{1}} \alpha_{1 i}^{2}
\end{aligned}
$$

From (29) and (30), we obtain (19). In a similar manner, (20)-(23) can be derived.

\section{REFERENCES}

[1] Mansell, A. R., and Bateman, A. (1994)

Practical implementation issues for adaptive predistortion transmitter linearization.

In IEE Colloq. Linear RF Amplifiers and Transmitters, Apr. 1994, London, 5/1-5/7.

[2] Dooley, S. R., and Nandi, A. K. (1998)

Adaptive time delay and frequency esitmation for digital signal synchronization in CDMA systems.

In Conference Record of the 32th Asilomar Conference Signals, Systems \& Computers, vol. 2, 1998, 1838-1842.

[3] Carter, G. C. (1993)

Coherence and Time Delay Estimation: An Applied Tutorial for Research, Development, Test and Evaluation Engineers. New York: IEEE Press, 1993.
[4] Itoh, K., Watanabe, S., Sato, T., and Hoshiko, Y. (1999) Radio source positioning using received time of arrival differences.

In Proceedings of IEEE Veh. Technology Conference, vol. 4, Sept. 1999, 2063-2066.

[5] Officier, C. B. (1958)

Introduction to the Theory of Sound Transmission, with Applications to the Ocean.

New York: McGraw-Hill, 1958.

[6] Sekizawa, S. (1998)

Estimation of arrival directions using MUSIC algorithm with a planar array.

In Proceedings of the IEEE International Conference

Universal Personal Communication, vol. 1, 1998, 555-559.

[7] Williams, R. E., and Battestin, H. F. (1971)

Coherent recombination of acoustic multipath signals propagated in the deep ocean.

Journal of the Acoustic Society of America, 50, 6, pt. 1 (1971), 1433-1442.

[8] Yuan, Y. X., Carter, G. C., and Salt, J. E. (2000)

Near-optimal range and depth estimation using a vertical array in a correlated multipath environment.

IEEE Transactioins on Signal Processing, 48, 2 (Feb. 2000), 317-330.

[9] Smith, J. O., and Friedlandler, B. (1985)

Adaptive multipath delay estimation.

IEEE Transactions on Acoustics, Speech, Signal Processing, 33, 4 (1985), 812-822.

[10] Ching, P. C., Chan, Y. T., and Ho, K. C. (1991)

Constrained adaptation for time delay estimation with multipath propagation.

IEE Proceedings, pt. F, 138, 5 (Oct. 1991), 453-458.

[11] Chan, Y. T., Riley, J. M. F., and Plant, J. B. (1981)

Modeling of time-delay and its application to estimation of nonstationary delays.

IEEE Transactions on Acoustics, Speech, Signal Processing, 29, 3 (1981), 577-581.

[12] Ching, P. C., and So, H. C. (1994)

Two adaptive algorithms for multipath time delay estimation.

IEEE Journal of Oceanic Engineering, 19, 3 (July 1994), 458-463.

[13] So, H. C., and Ching, P. C. (1996)

A generalized equalization-based algorithm for multipath time delay estimation.

In Proceedings of the IEEE International Symposium on Circuits and Systems, vol. 2, Atlanta, GA, May 1996, 556-559.

[14] Widrow, B. et al. (1976)

Stationary and nonstationary learning characteristics of the LMS adaptive filter.

Proceedings of the IEEE, 64, 8 (1976), 1151-1162.

[15] Oppenheim, A. V., and Schafer, R. W. (1975)

Digital Signal Processing.

Englewood Cliffs, NJ: Prentice-Hall, 1975.

[16] So, H. C., Ching, P. C., and Chan, Y. T. (1994)

A new algorithm for explicit adaptation of time delay. IEEE Transactions on Signal Processing, 42 (July 1994), $1816-1820$. 


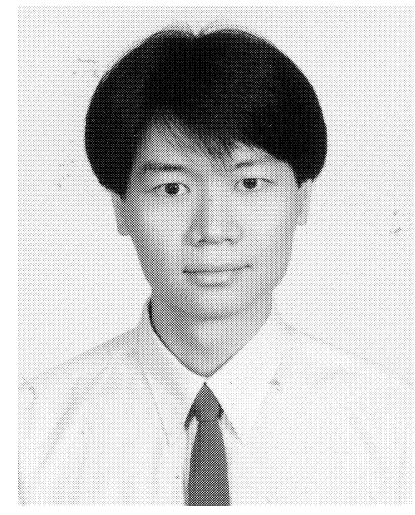

H. C. So (S'90-M'96) was born in Hong Kong. He received the B.Eng. degree from City Polytechnic of Hong Kong and the Ph.D. degree from the Chinese University of Hong Kong, both in electronic engineering, in 1990 and 1995, respectively.

From 1990 to 1991, he was an electronic engineer at the Research \& Development Division of Everex Systems Engineering Ltd. During 1995-1996, he worked as a Post-Doctoral Fellow at the Chinese University of Hong Kong. From 1996 to 1999, he was a research assistant professor at the Department of Electronic Engineering, City University of Hong Kong. Currently he is an assistant professor in the Department of Computer Engineering and Information Technology at City University. His research interests include adaptive filter theory, detection and estimation, wavelet transform, and signal processing for communications and multimedia. 